\title{
»Deliberative Polling « als Methode zum Erlernen des demokratischen Sprechens
}

\section{Einfübrung}

Eine Grundthese der in den letzten Jahren stark gewachsenen Literatur zur deliberativen Demokratie ist, dass die Stabilität und die Qualität einer Demokratie nicht nur von formalen Institutionen, wie einem Wahlsystem oder der Struktur parlamentarischer Repräsentation, abhängen, sondern ganz entscheidend auch von bestimmten demokratischen Kompetenzen der Bürger, insbesondere dem demokratischen Kommunikationsverhalten. Nach dieser These gehört zu den zentralen Kompetenzen zur Aufrechterhaltung und Entwicklung der Demokratie vor allem die Fähigkeit zur demokratischen Deliberation, das heißt zur Argumentation, zur Abwägung und schließlich zur Entscheidung über alternative Problemlösungen².

1 C. List, London School of Economics, Department of Government, London WC2A 2AE, U.K.; c.list@lse.ac.uk. A. Sliwka, Universität Erfurt, Institut für Allgemeine Erziehungswissenschaft und Empirische Bildungsforschung, 99105 Erfurt; anne.sliwka @uni-erfurt.de.

Wir danken sehr herzlich den Teilnehmern der Arbeitsgruppe »Demokratie und Deliberation « auf der Schülerakademie Gaesdonck 2002, ohne deren engagierte Mitarbeit das hier vorgestellte Projekt nicht hätte durchgeführt werden können: Laura Birg, Mira Colsmann, Florian Frederico Cortez Kiesow, Anne-Marie Dörnenburg, Andreas Englberger, Tanja Greiner, Corinna Gundelach, Irene Köppe, Fabian Löffler, Frieder Meidert, Richard Peter, Anne Rittstieg, Armin Schmidt, Tim Stoffel, Ulrike Zirpel. Wir danken der Deutschen SchülerAkademie in Bonn für die Ermöglichung des Projekts und allen anderen Teilnehmern und Mitarbeitern der Akademie Gaesdonck 2002 für ihre Teilnahme an unseren Umfragen und den Deliberationsveranstaltungen. Für sehr hilfreiche Hinweise danken wir Volker Brandt und James Fishkin. Die Begriffe »Deliberative Polling « und »Deliberative Poll« sind in den USA von James Fishkin als $»$ trademark $(\mathrm{TM}) \ll$ registriert worden.

2 Zur Theorie der deliberativen Demokratie siehe unter vielen anderen J. Bohman / W. Rehg (Hg.), Deliberative Democracy: Essays on Reason and Politics, Cambridge, MA 1997; J. Cohen, "Deliberation and Democratic Legitimacy « in: A. Hamlin / P. Pettit (Hg.), The Good Polity: Normative Analysis of the State, Oxford 1989, S. 17-34; J. S. Dryzek, Discursive Democracy: Politics, Policy and Political Science, New York 1990; J. S. Dryzek, Deliberative Democracy and Beyond, Oxford 2000; J. Elster, »Introduction « in: J. Elster (Hg.), Deliberative Democracy, New York 1998, S. 1-18; A. Gutmann / D. Thompson, Democracy and Disagreement, Cambridge, MA 1996; M. Becker, »Politik als Verständigungsprozess Modelle deliberativer Demokratie« in: Zeitschrift für Politik, 2. Heft, 2000. 
Selbst der Zusammenbruch einer Demokratie ist aus dieser Sicht nicht immer nur zurückzuführen auf das Versagen demokratischer Institutionen, sondern kann auch eine Folge der Abwesenheit demokratischer Einstellungen oder Kommunikationsformen sein. So wird manchmal argumentiert, dass die zusammenbrechende Weimarer Republik der 1920er und 1930er Jahre eine »Demokratie ohne Demokraten« war.

Vertritt man diese Grundthese und betrachtet man die Förderung demokratischer Kompetenzen als (normativ) wünschenswert, so stellt sich die (empirische und pragmatische) Frage, welche Mittel sich zur Förderung dieser Kompetenzen in der Gesellschaft eignen. Diese Frage steht unter anderem im Mittelpunkt der Debatte zur Demokratieerziehung (»civic education «): Welche Rolle können und sollen Bildungszusammenhänge (z. B. Schule und Hochschule) für die Förderung demokratischer Kompetenzen spielen?

In dieser Arbeit stellen wir die von James Fishkin entwickelte Methode des Deliberative Polling vor und zeigen, dass sich diese Methode zur Förderung demokratischer Kompetenzen in Bildungszusammenhängen anwenden lässt ${ }^{3}$. Wir berichten von einem am Deliberative Polling orientierten Projekt, das wir in einem Bildungszusammenhang (nämlich auf einer sogenannten Schülerakademie, wie unten beschrieben) durchgeführt haben, und diskutieren die Ergebnisse vor dem Hintergrund verschiedener theoretischer Kriterien. Es zeigt sich, dass sich das Deliberative Polling - über seine qualitative Bedeutung für das Erlernen demokratischer Kommunikation hinaus - auf zwei wichtige quantitative Kriterien positiv auswirken kann: erstens auf den Informationsstand der Teilnehmer und zweitens auf die kollektive Kobäsion der Präferenzen unterschiedlicher Teilnehmer. Beide Kriterien werden unten näher erläutert.

Die Arbeit ist wie folgt aufgebaut. Im Abschnitt 2 gehen wir kurz auf den theoretischen Hintergrund ein, nämlich die Kontroverse zwischen sogenannten aggregativen und deliberativen Demokratiemodellen. Im Abschnitt 3 besprechen wir verschiedene existierende Ansätze zum Erlernen des demokratischen Sprechens. Im Abschnitt 4 erläutern wir die Methode des Deliberative Polling. Im Abschnitt 5 berichten wir über das von uns durchgeführte Deliberationsprojekt und werten die Ergebnisse des Projekts aus. Im Abschnitt 6 schließlich ziehen wir Schlussfolgerungen.

\section{Aggregative und deliberative Demokratiemodelle}

Die gegenwärtige politikwissenschaftliche Diskussion zur Demokratie wird bestimmt von zwei entgegengesetzten Modellen, dem Modell der aggregativen Demokratie und dem der deliberativen Demokratie.

Das aggregative Demokratiemodell stellt das Wählen oder Abstimmen zwischen konfliktträchtigen individuellen Präferenzen in den Mittelpunkt und modelliert De-

3 Siehe J. S. Fishkin, Democracy and Deliberation, New Haven/London 1991; J. S. Fishkin, The Voice of the People, New Haven/London 1995. 
mokratie als »Input-Output-System«. Demokratie besteht nach dieser Vorstellung in dem mechanischen Zusammenfassen (im Aggregieren) individueller Inputs (z. B. individueller Präferenzen oder Wählerstimmen) zu kollektiven Outputs (z. B. kollektive Präferenzen oder kollektive Entscheidungen). Die zentrale demokratische Institution ist das verwendete Wahl- oder Aggregationsverfahren. Als Aggregationsverfabren bezeichnet man einen Mechanismus, der eine Kombination von individuellen Präferenzordnungen in eine einzige kollektive Präferenzordnung überführt ${ }^{4}$. Die Qualität einer Demokratie ist aus Sicht des aggregativen Demokratiemodells in starkem Maße abhängig von der Qualität des verwendeten Aggregationsverfahrens.

Das deliberative Demokratiemodell hingegen stellt nicht das mechanische Aggregieren von Individualpräferenzen zu Kollektivpräferenzen in den Mittelpunkt, sondern das demokratische Sprechen (die Deliberation) über diese Präferenzen. Demokratie wird modelliert als kommunikatives System, als die Gesamtheit derjenigen gesellschaftlichen Meinungsbildungs-, Lern- und Handlungsprozesse, die zu kollektiven Entscheidungen führen. Die Qualität einer Demokratie ist daher abhängig von der Qualität dieser Prozesse.

\subsection{Die Problematik der Aggregation: Condorcets Paradoxon und Arrows Unmöglichkeitssatz}

Dass die Aggregation von pluralistischen Individualpräferenzen ein nicht-triviales Problem darstellt, macht das Condorcet-Paradoxon deutlich ${ }^{5}$. Das Paradoxon zeigt, dass das plausible Aggregationsverfahren der paarweisen Mehrbeitswabl zu inkonsistenten kollektiven Präferenzen führen kann. Wir betrachten eine Situation, in der drei Individuen (Wähler 1, 2, 3) eine kollektive Entscheidung über drei Alternativen (Kandidaten $x, y, z)$ treffen müssen. Die Individuen (Wähler) haben folgende Präferenzen (das Symbol » « wird interpretiert als »wird präferiert gegenüber«):

Individuum 1: $\quad x>y>z$

Individuum 2: $\quad y>z>x$

Individuum 3: $\quad z>x>y$

Die Kollektivpräferenz soll nun durch Mehrheitsvergleiche zwischen jeweils zwei Alternativen ermittelt werden: Eine Mehrheit von 2 aus 3 (bestehend aus Individuen 1 und 3) präferiert $x$ gegenüber $y$, eine weitere Mehrheit von 2 aus 3 (bestehend aus Individuen 1 und 2) präferiert $y$ gegenüber $z$, und eine dritte Mehrheit von 2 aus 3 (bestehend aus Individuen 2 und 3) präferiert $z$ gegenüber $x$. Die kollektive Präferenzordnung ist daher $x>y>z>x$, eine »zyklische« und somit inkonsistente Präferenzordnung. Insbesondere existiert kein Condorcet-Sieger. Als Condorcet-Sieger

4 Im mathematischen Modell werden die Individuen mit den Ziffern 1, 2, .., $n$ bezeichnet. Jedem Individuum, $i$, wird genau eine Präferenzordnung, $P_{i}$, über eine Menge an Alternativen, $x, y, z, \ldots$, zugeordnet. Ein Aggregationsverfabren ist eine Funktion, $F$, die jeden Vektor von Präferenzordnungen, $<P_{1}, P_{2}, \ldots, P_{n}>$ (bestehend aus genau einer Präferenzordnung für jedes Individuum), in eine einzige kollektive Präferenzordnung, $P$, überführt.

5 Siehe I. McLean / F. Hewitt (Hg.), Condorcet: Foundations of Social Choice and Political Theory, Cheltenham 1994. 
bezeichnet man eine Alternative, die gegenüber jeder anderen Alternative von einer Mehrheit (oder mindestens der Hälfte) der Individuen präferiert wird. Das Condorcet-Sieger-Kriterium wird von vielen Politikwissenschaftlern als eine besonders plausible Formalisierung des Kriteriums des Allgemein- oder Mehrheitswillens angesehen ${ }^{6}$.

Ist das Condorcet-Paradoxon das Resultat eines künstlichen Gedankenexperiments, oder ist es ein Anzeichen eines grundsätzlicheren Problems, das bei der Aggregation gegensätzlicher individueller Präferenzen entstehen kann? Das Condorcet-Paradoxon selbst zeigt nur, dass ein bestimmtes plausibles Aggregationsverfahren, nämlich die paarweise Mehrheitswahl, zu inkonsistenten kollektiven Präferenzen führen kann. Das Paradoxon macht jedoch keine Aussage darüber, ob dieses Problem möglicherweise mit Hilfe eines anderen, ebenso plausiblen Aggregationsverfahrens zu lösen ist. Arrows Unmöglichkeitssatz behandelt diese Frage $^{7}$. Stellt man fünf plausible Minimalbedingungen auf, die ein Aggregationsverfahren erfüllen soll, so zeigt Arrows Satz, dass kein Aggregationsverfahren existiert, das diese Bedingungen gleichzeitig erfüllt. Die Bedingungen, die erfüllt werden sollen, sind:

Unbeschränkte Definitionsmenge (U): Jede logisch mögliche Kombination von individuellen Präferenzen ist als Input des Aggregationsverfahrens zulässig.

Transitivität (T): Wenn $x$ gegenüber $y$ kollektiv präferiert wird und $y$ gegenüber $z$, dann wird auch $x$ gegenüber $z$ kollektiv präferiert.

Das schwache Pareto-Prinzip $(P)$ : Wenn alle Individuen $x$ gegenüber $y$ präferieren, dann wird $x$ gegenüber $y$ auch kollektiv präferiert.

Nicht-Diktatur (D): Es existiert kein Individuum (ein »Diktator «), das für alle Kombinationen von individuellen Präferenzen immer die kollektive Präferenz bestimmt.

Unabhängigkeit von irrelevanten Alternativen (IIA): Die kollektive Präferenz zwischen zwei Alternativen $x$ und $y$ hängt nur von den individuellen Präferenzen zwischen $x$ und $y \mathrm{ab}$, nicht jedoch von den individuellen Präferenzen über andere Alternativen.

Diese Bedingungen werden als minimal angesehen, weil man im Idealfall noch anspruchsvollere Bedingungen von einem Aggregationsverfahren erfüllt sehen möchte ${ }^{8}$.

6 Siehe z. B. D. Estlund / J. Waldron / B. Grofman / S. Feld, »Democratic Theory and the Public Interest; Condorcet and Rousseau Revisited « in: American Political Science Review 83, 1989, S. 1317-1340.

7 Siehe K. Arrow, Social Choice and Individual Values, New York 1951.

8 Solche Bedingungen sind z. B. die sogenannte Bedingung der Anonymität $(A)$, die besagt (informell), dass die Präferenzordnungen aller Individuen das gleiche Gewicht in der Aggregation haben sollen, sowie die Bedingung des starken Pareto-Prinzips (StP), die besagt, dass $x$ gegenüber $y$ kollektiv präferiert werden soll, wenn kein Individuum $y$ gegenüber $x$ präferiert und mindestens ein Individuum $x$ gegenüber $y$ präferiert. Diese Bedingungen sind im folgenden logischen Sinne anspruchsvoller als Arrows Bedingungen: Bedingung (A) impliziert Bedingung (D), nicht jedoch umgekehrt; und Bedingung $(\mathrm{StP})$ impliziert Bedingung $(\mathrm{P})$, nicht jedoch umgekehrt. 
Satz (Arrows Unmöglichkeitssatz): Es existiert kein Aggregationsverfahren, das die Bedingungen (T), (U), (P), (D) und (IIA) erfüllt.

Aus Arrows Satz folgt, dass jedes Aggregationsverfahren notwendigerweise mindestens eine der Arrowschen Bedingungen verletzt.

\subsection{Deliberation und Konsens}

Die Lösbarkeit von Aggregationsproblemen ist vor allem davon abhängig, wie unterschiedlich die Präferenzen der verschiedenen Individuen sind. Es ist offensichtlich, dass im Fall vollständiger Einstimmigkeit aller Individualpräferenzen die Aggregation dieser Präferenzen keine Schwierigkeiten aufwirft.

Eine Hypothese, die von frühen Befürwortern des deliberativen Demokratiekonzepts oft vertreten wurde, besagt, dass eine hinreichende Phase der Deliberation individuelle Präferenzen so verändern kann, dass es zu einem vollständigen Konsens (Einstimmigkeit der Präferenzen) und damit zur Vermeidung von Condorcet-Paradoxien und zur Auflösung des Arrowschen Aggregationsproblems kommt.

Jon Elster z. B. fasst diese Hypothese folgendermaßen zusammen: »The core of the theory [of deliberative democracy] ... is that rather than aggregating or filtering preferences, the political system should be set up with a view to changing them by public debate and confrontation. The input to the social choice mechanism would then not be the raw, quite possibly selfish or irrational preferences ..., but informed and other-regarding preferences. Or rather, there would not be any need for an aggregation mechanism, since a rational discussion would tend to produce unanimous preferences. «9

Unabhängig davon, ob ein vollständiger Konsens wünschenswert ist oder nicht, existiert kein empirischer Hinweis dafür, dass Deliberation im Normalfall einen solchen Konsens hervorbringen kann. Die Hypothese, Deliberation könne das Problem der demokratischen Aggregation durch vollständige Konsensfindung auflösen, scheint daher empirisch und pragmatisch fragwürdig.

\subsection{Deliberation und Metakonsens}

Eine weniger anspruchsvolle, aber dennoch nicht triviale Hypothese besagt, dass Deliberation im Normalfall zwar nicht einen vollständigen Konsens hervorbringen kann, jedoch einen Metakonsens ${ }^{10}$. Von einem Metakonsens spricht man, wenn die Individuen über die dem Entscheidungsproblem zugrunde liegende ideologische

9 Siehe J. Elster, »The Market and the Forum « in: J. Elster / A. Hylland (Hg.), Foundations of Social Choice Theory, Cambridge 1986, S. 103-132.

10 Verschiedene Versionen dieser Hypothese sind $\mathrm{zu}$ finden in D. Miller, »Deliberation and Social Choice in: Political Studies (Sonderausgabe) 40, 1991, S. 54-67; J. Knight / J. Johnson, »Aggregation and Deliberation: On the Possibility of Democratic Legitimacy « in: Political Theory 22, 1994, S. 277-296; J. S. Dryzek / C. List, »Social Choice Theory and Deliberative Democracy: A Reconciliation « in: British Journal of Political Science 33, 2003, S. 1-28. 
Dimension übereinstimmen und sich die individuellen Präferenzen aller Individuen auf derselben Links-Rechts-Achse systematisch anordnen lassen. Dies fordert nicht einen vollständigen Konsens in Form der Übereinstimmung der individuellen Präferenzen. In einem politischen Beispiel könnte ein Metakonsens etwa darin bestehen, dass alle Individuen über die ideologische Links-Rechts-Anordnung der politischen Parteien übereinstimmen (z. B. in der Reihenfolge PDS, Grüne, SPD, FDP, CDU, CSU), ohne sich darüber zu einigen, welche Partei am meisten präferiert wird. Die Individuen stimmen in der Strukturierung des Entscheidungsproblems überein, nicht jedoch notwendigerweise in der bevorzugten Lösung.

Man bezeichnet eine Kombination (im mathematischen Modell einen Vektor) von individuellen Präferenzordnungen als eingipflig (single-peaked), wenn mindestens eine Links-Rechts-Anordnung der Alternativen (Kandidaten) existiert, so dass jedes Individuum (jeder Wähler) eine Maximalpräferenz auf dieser Links-RechtsAchse hat und Alternativen (Kandidaten) umso weniger präferiert, je weiter sie entfernt sind von der Position der Maximalpräferenz auf der Achse.

Darstellung 1 zeigt ein Beispiel zweier Präferenzordnungen, die eingipflig sind in Bezug auf dieselbe Links-Rechts-Achse (nämlich die Achse $x-z-v-y-w)$.

\section{Darstellung 1}

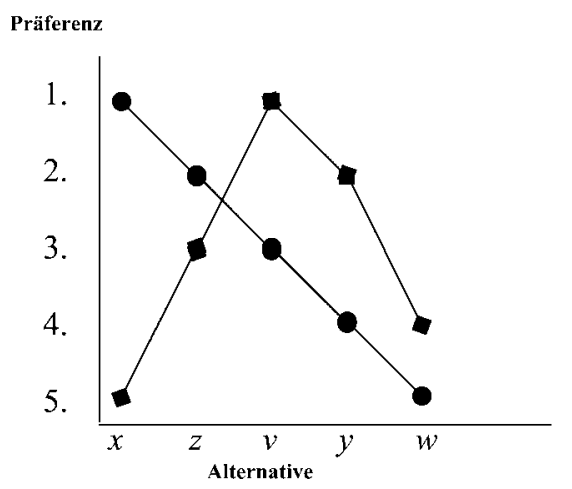

Erfüllen die Präferenzordnungen aller Individuen die Eigenschaft der Eingipfligkeit in Bezug auf dieselbe Links-Rechts-Anordnung der Alternativen (Kandidaten), so kann dies ein Indiz eines Metakonsenses sein ${ }^{11}$.

11 Siehe C. List, »Two Concepts of Agreement« in: The Good Society 11, 2002, S. 72-79. 
Die Bedeutung des Kriteriums der Eingipfligkeit (und damit des Metakonsenses) liegt in der Tatsache, dass Eingipfligkeit hinreichend zur Vermeidung von Condorcet-Paradoxien und des Arrowschen Aggregationsproblems ist ${ }^{12}$.

Satz (Satz von Black): Sind die Präferenzen aller Individuen eingipflig in Bezug auf dieselbe Links-Rechts-Anordnung der Alternativen, so existiert immer ein Condorcet-Sieger (wie oben definiert) ${ }^{13}$.

Ersetzt man Arrows Bedingung der unbeschränkten Definitionsmenge $(U)$ durch die schwächere Bedingung der Definitionsmenge eingipfliger Präferenzen (SP), so gelangt man zu einer Möglichkeitsaussage im Gegensatz zu der Arrowschen Unmöglichkeitsaussage.

Definitionsmenge eingipfliger Präferenzen (SP): Als Input des Aggregationsverfahrens ist zulässig jede Kombination von individuellen Präferenzen, die die Eigenschaft der Eingipfligkeit in Bezug auf dieselbe Links-Rechts-Achse erfüllt.

Satz: Es existiert ein Aggregationsverfahren, das die Bedingungen (SP), (T), (P), (D) und (IIA) erfüllt, nämlich das paarweise Mehrheitswählen ${ }^{14}$.

Man kann über die Resultate von Black und Arrow hinaus zeigen, dass auch ein hohes Maß an partieller Eingipfligkeit schon hinreichend sein kann für das Vermeiden von Aggregationsparadoxien ${ }^{15}$. Eine Situation partieller Eingipfligkeit liegt dann vor, wenn die Präferenzordnungen einer Teilmenge der Individuen die Eigenschaft der Eingipfligkeit in Bezug auf dieselbe Links-Rechts-Anordnung der Alternativen erfüllen.

Wir werden in der Diskussion des Deliberative Polling unten näher auf die Frage eingehen, ob sich die Hypothese, Deliberation könne einen Metakonsens hervorbringen, empirisch bestätigen lässt. Die theoretischen Überlegungen dieses Abschnitts geben uns jedoch schon einen ersten Grund zu der Annahme, dass Deliberationsprozesse möglicherweise von Relevanz sein können für die Lösbarkeit von Problemen der Aggregation. Der Schlüssel zu einer deliberativen Lösung von Aggregationsproblemen liegt jedoch nicht - wie von frühen deliberativen Demokratiebefürwortern angenommen - in einem durch Deliberation hervorgebrachten vollständigen Konsens, sondern eher in einem durch Deliberation hervorgebrachten Metakonsens.

12 Zur technischen Vereinfachung (das heisst zur Vermeidung harmloser Mehrheitspattsituationen) kann man im mathematischen Modell annehmen, dass die Anzahl der Individuen, $n$, ungerade ist.

13 Siehe D. Black, »On the Rationale of Group Decision-Making « in: Journal of Political Economy, 56, 1948.

14 Ebd.; Arrow, aaO. (FN 7).

15 Siehe R. G. Niemi, »Majority Decision-Making with Partial Unidimensionality in: American Political Science Review 63, 1969, S. 488-497. 


\section{Bestehende Ansätze zum Erlernen des demokratischen Sprechens in Bildungszusammenbängen}

Deliberation bedeutet vernunftgeleitetes und freies Sprechen mit dem Ziel einer schrittweisen Verständigung über die Präferenzen einzelner beteiligter Individuen. Wie schon oben erläutert, wird Deliberation oft mit der Begründung vertreten, dass das behutsame und vernünftige Sprechen die Suche nach einer für alle Beteiligten tragfähigen Lösung, also einem Konsens, oder zumindest nach einer gemeinsamen Problemstrukturierung, also einem Metakonsens, erleichtern würde.

Bildungszusammenhänge (z. B. Schule und Hochschule) bieten derzeit wenig Möglichkeiten zum Erfahren von demokratischer Deliberation. Einfache didaktische Formen wie das kooperative Lernen und die Mediation enthalten Elemente des deliberativen Sprechens. Angesichts der Kompetenzerfordernisse komplexer demokratischer Prozesse dienen solche vergleichsweise einfachen Methoden allerdings bestenfalls zur Einführung sehr junger Lernender. Die Entwicklung demokratischer Kompetenzen bei älteren Schülern und Erwachsenen bedarf neuer anspruchsvoller Methoden.

\subsection{Das »Debating«}

Das in Bildungszusammenhängen vor allem im angelsächsischen Raum durchgängig vertretene »Debating « ist dialektisch angelegt. Zunächst werden dabei bewusst eine Frontstellung festgelegter, unterschiedlicher Positionen hergestellt und Extrempositionen rhetorisch ausgereizt. Nach der Debatte findet eine Abstimmung statt. Deliberation unterscheidet sich jedoch deutlich von einer in eine Abstimmung mündenden Debatte. Deliberatives Sprechen zeichnet sich dadurch aus, dass Teilnehmer an einem solchen Sprechakt von ihrer eigenen Position zurücktreten und sich grundlegender auf eine gemeinsame Analyse eines Problemfeldes einlassen, die alle vorhergehenden Einzelpositionen in analytischer Weise reflektiert, um die den Positionen zugrunde liegenden mentalen Modelle freizulegen. Deliberative Verständigungsprozesse überwinden das Bestreben zu rhetorischer Brillanz oder Schlagfertigkeit, das die Form der Debatte auszeichnet, zugunsten einer kleinschrittigen Suche nach grundlegender Verständigung.

\subsection{Parlamentssimulationen}

Die Kommunikationsprozesse, die parlamentarischen Meinungsbildungs- und Entscheidungsverfahren zugrunde liegen, sind in ihrer Komplexität didaktisch schwer vermittelbar und für Lernende entsprechend schwer nachvollziehbar. Ein verständnisintensives Lernen solcher Prozesse lässt sich durch die Umsetzung von Simulationen erreichen, die reale demokratische Prozesse abzubilden versuchen. In Parlamentssimulationen wie dem an amerikanischen High Schools umgesetzten »Congress in Action « oder dem inzwischen international verbreiteten "Model United Nations « werden Schüler in einen demokratischen »Mikrokosmos« versetzt, in dem 
sie die Rolle eines Handelnden in einem politischen Prozess übernehmen und über einen längeren Zeitraum ausfüllen.

Das Programm »Congress in Action « beispielsweise wird an US-amerikanischen High Schools mit Schülern der Jahrgangsstufen 11 oder 12 durchgeführt. Die Simulation des Kongresses läuft über einen Zeitraum von mehreren Wochen. Die Lernenden übernehmen für diesen Zeitraum die Rolle eines Abgeordneten bzw. Kabinettsmitglieds. Anhand von authentischem Material zu der Funktion, den Interessenschwerpunkten, den Überzeugungen und Loyalitäten sowie den Netzwerken dieses Abgeordneten bereiten sie sich auf die Rolle vor. Jeder Lernende ist in eine bestimmte Gesetzgebungsinitiative eingebunden und muss in seiner Abgeordnetenrolle dazu ein wissensgestütztes Positionspapier erarbeiten. Auf der Grundlage dieser Position arbeitet der Lernende auch in simulierten Ausschusssitzungen sowie Plenardebatten mit, in denen er seine Position vertritt. Alle Entscheidungs- und Abstimmungsprozesse eines realen Parlamentes werden dabei auf der Grundlage tatsächlicher Mechanismen und Funktionsweisen des US-Kongresses simuliert. Die pädagogische Funktion dieses aufwendigen Lernarrangements liegt in der Ermöglichung eines anschaulichen Verstehens realer politischer Prozesse in der parlamentarischen Demokratie.

\subsection{Das »National Issues Forum«}

Aufgrund ihrer meist nur punktuellen Beteiligung (z. B. bei Wahlen) in modernen Demokratien sind Bürger über gesellschaftspolitische Probleme oft nur begrenzt informiert. Gerade in der Mediendemokratie scheinen politische Meinungsbilder im Fluss und von den jeweils dominierenden Schlagzeilen geprägt. Eine Demokratie, die Bürgern wenig eigene Entscheidungsfindung abverlangt, lässt die Investition von Zeit und Mühe in eine differenzierte Auseinandersetzung mit politischer Information nicht lohnenswert erscheinen - eine solche Demokratie erzeugt nach Aussage von Politikwissenschaftlern einen weitverbreiteten Zustand »rationaler Ignoranz $\ll^{16}$.

Eine gezielt für die Demokratieerziehung entwickelte Methode stellt das National Issues Forum dar, das zur Einübung von Deliberationsfähigkeit beitragen soll17. Nach dem Modell der »New England Town Meetings«, einer der tragenden Gründungsmetaphern der amerikanischen Demokratie, bringt das National Issues Forum Bürger zusammen, die über spezifische Themen wie Drogenmissbrauch, soziale Sicherungssysteme, Arbeitsmarktpolitik oder Jugendkriminalität deliberieren. National Issues Foren finden in Schulen und Hochschulen statt, werden jedoch auch von Kommunen, kirchlichen Gruppen oder anderen zivilgesellschaftlichen Assoziationen veranstaltet. Ziel der Foren ist es, Menschen mit unterschiedlichen weltanschaulichen Ausrichtungen die Möglichkeit zu geben, durch Deliberation auf

16 Siehe B. Caplan, »Rational Ignorance versus Rational Irrationality« in: Kyklos 54(1), 2001, S. 3-26.

17 Siehe http://www.nifi.org/. 
der Grundlage von Information gemeinsame Ziele für politisches Handeln zu finden und zu formulieren.

National Issues Foren sind strukturierte Diskussionen, die von ausgebildeten Moderatoren geleitet werden. Die Teilnehmer müssen keinerlei Expertise mitbringen, sondern erhalten zu ihrer Information vor Beginn des Forums umfangreiches Arbeitsmaterial. Auf der Grundlage umfassender und möglichst neutraler Informationen wägen die Teilnehmer an einem National Issues Forum unterschiedliche politische Lösungen zu einem Problem gegeneinander ab. Sie analysieren die unterschiedlichen Alternativen und die dazugehörigen Pro- und Kontra-Argumente. Dabei versuchen sie - angeleitet durch Moderatoren - sowohl ihre eigene Perspektive als auch die des Gemeinwohls in Betracht zu ziehen. Die Foren haben keine unmittelbare bindende Wirkung auf die Politik, doch Ergebnisse werden an lokale und nationale Politiker weitergeleitet und mit diesen diskutiert. Oft ist ein National Issues Forum auch eine Grundlage für das weitere Handeln von Bürgern in einer Gemeinde.

\section{Das Deliberative Polling}

Eine Methode, deren Anwendungsmöglichkeiten in der Demokratieerziehung (»civic education «) noch nicht hinreichend erforscht ist, ist das von James Fishkin entwickelte Deliberative Polling ${ }^{18}$. Die Methode des Deliberative Polling hat aggregative wie auch deliberative Komponenten.

In Deliberative Polls wird ein kontroverses Thema ausgewählt. Zunächst wird eine Gruppe von ca. 100-350 Testpersonen (typischerweise eine repräsentative Stichprobe aus der Bevölkerung) mit Hilfe von Fragebögen einzeln zu diesem Thema befragt.

Der zweite Schritt ist eine Phase der Deliberation. Die Testpersonen werden eingeladen, ein bis drei Tage lang über die Thematik dieser Meinungsumfrage in deliberativer Form zu beraten. Zu ihrer Vorbereitung erhalten die Teilnehmer ausgewogenes Informationsmaterial, das zur allgemeinen Kontrolle auch öffentlich zugänglich gemacht wird. Die Deliberationsphase setzt sich nun aus mehreren Arbeitsschritten zusammen. In großen Plenarsitzungen stellen unterschiedliche Experten und Interessenvertreter (wie auch Politiker) die verschiedenen Positionen zu der Thematik vor. In kleineren Diskussionsgruppen unter der Anleitung von Moderatoren entwickeln die Teilnehmer Fragen, die sie dann wiederum innerhalb einer großen Plenarsitzung an die Experten und Interessenvertreter stellen und mit ihnen diskutieren.

Abschließend wird jeder Teilnehmer erneut befragt unter Verwendung desselben Fragebogens wie in der ersten Meinungsumfrage. Man kann dann die individuellen

18 Siehe Fishkin, Democracy and Deliberation, aaO. (FN 3); Fishkin, The Voice of the People, aaO. (FN 3); R. C. Luskin / J. S. Fishkin / R. Jowell, »Considered Opinions: Deliberative Polling in Britain « in: British Journal of Political Science 32(3), 2002, S. 455-487. 
Meinungen und Präferenzen vor der Deliberation mit denen nach der Deliberation vergleichen.

Deliberative Polls sind schon zu verschiedenen Themen und in verschiedenen Ländern durchgeführt worden, z. B. zur Energieversorgung in Texas, zur Kriminalität in Großbritannien, zur Frage nach dem Beitritt zum Euro in Dänemark und zum Verfassungsreferendum zur Abschaffung der Monarchie in Australien. Fishkin und seine Kollegen konnten häufig nennenswerte Meinungsverschiebungen nachweisen. Auch konnten sie zeigen, dass öffentliche Interessen im Gegensatz zu Einzelinteressen im Meinungsbild nach der Phase der Deliberation oft stärker in den Vordergrund rücken. Darüber hinaus enthalten die Fragebögen in einigen Deliberative Polls Fragen, die Aufschluss über den Informationsstand der Befragten geben, etwa faktische Wissensfragen. Die Auswertung solcher Fragen konnte einen erhöhten Informationsstand nach der Deliberationsphase nachweisen.

Deliberative Polls eignen sich zur Überprüfung der oben eingeführten Hypothese, dass Deliberation einen Metakonsens hervorbringen kann. Daten aus Deliberative Polls haben gezeigt, dass Deliberation zwar typischerweise nicht vollständige Eingipfligkeit (wie oben definiert), jedoch ein hohes Maß an partieller Eingipfligkeit hervorrufen kann ${ }^{19}$.

Auch wenn sich das Forschungsinteresse von Fishkin und seinen Kollegen eher auf die empirischen Daten bezieht, die durch das Verfahren generiert werden, so ist das Deliberative Polling doch, wie Fishkin sagt, »both a social science experiment and a form of public education in the broadest sense $\aleph^{20}$. Der letztere Aspekt des Deliberative Polling steht bei dem hier vorgestellten Deliberationsprojekt im Vordergrund. Im Gegensatz zu den im vergangengen Abschnitt vorgestellten Ansätzen zur Demokratieerziehung bietet das Deliberative Polling jedoch eine Möglichkeit der quantitativen Evaluation des Deliberationsprozesses mit Hilfe der vor und nach der Deliberation generierten Umfragedaten.

\section{Ein Deliberationsprojekt in einem Bildungszusammenhang}

Wir führten ein am Deliberative Polling orientiertes Projekt im Rahmen einer $2^{1} / 2^{-}$ wöchigen Veranstaltung der Deutschen Schülerakademie durch ${ }^{21}$. Die Akademie

19 Siehe C. List / I. McLean / J. S. Fishkin / R. Luskin, »Can Deliberation Increase Preference Structuration? Evidence from Deliberative Polls«, Conference of the American Political Science Association, Washington, DC 2000.

20 Siehe http://www.law.utexas.edu/research/delpol/index.html.

21 Der mit Mitteln des Bundesministeriums für Bildung und Forschung geförderte Verein Bildung und Begabung e. V. führt jährlich im Sommer mehrere Akademien für begabte Schüler der gymnasialen Oberstufe durch. Jede Akademie setzt sich aus mehreren Arbeitsgruppen zu unterschiedlichen Themen zusammen. Jeder Akademieteilnehmer ist einer bestimmten Arbeitsgruppe zugeordnet, hat jedoch die Möglichkeit zur Teilnahme an einem akademieübergreifenden Rahmenprogramm. In der Akademie werden die Schüler mit Formen des wissenschaftlichen Arbeitens vertraut gemacht. 
bestand aus 6 Arbeitsgruppen mit jeweils ca. 15 Teilnehmern. Die von uns geleitete Arbeitsgruppe trug den Titel »Demokratie und Deliberation: vom Umgang mit Konflikten in einer pluralistischen Gesellschaft «. Unsere Arbeitsgruppe hatte ein theoretisches und ein anwendungsorientiertes Ziel. Das theoretische Ziel war, die Kontroverse zwischen aggregativen und deliberativen Demokratiemodellen zu erarbeiten und zu diskutieren. Arbeitsmethoden waren hier Textarbeit, kurze Referate und Diskussionen. Das anwendungsorientierte Ziel war, gemeinsam mit den Teilnehmern unserer Arbeitsgruppe eine konkrete Deliberationsumfrage zu planen, durchzuführen und auszuwerten. Das Thema war »Aktuelle Fragen der Bildungspolitik in Deutschland «. Die Testpersonen waren die Akademieteilnehmer und -dozenten der anderen Arbeitsgruppen (insgesamt 94). Die Gruppe der Befragten war damit nicht wie in Fishkins Deliberative Polls eine repräsentative Stichprobe aus der Referenzbevölkerung. Der von unserer Arbeitsgruppe entwickelte Fragebogen ist im Anhang abgedruckt. Der Fragebogen enthält Fragen zu Studienfinanzierung, Hochschulzugang, Abiturprüfungen, Struktur des Schulsystems (Gesamtschule versus dreigliedriges System), Halbtags- versus Ganztagsschulen.

Es fand zunächst eine Erstbefragung der Testpersonen statt, dann eine Deliberationsphase, bestehend aus zwei abendlichen Veranstaltungen (mit einer Beteiligung von 93 bzw. 85 Personen), und schließlich eine Zweitbefragung. Die Deliberationsphase orientierte sich an der von Fishkin entwickelten Struktur. Zur Information der Befragten verteilten wir nach der Erstbefragung von unserer Arbeitsgruppe entwickelte Informationsmaterialien mit Pro- und Kontra-Argumenten zu den einzelnen Standpunkten. Die erste, zeitlich etwas längere Abendveranstaltung bezog sich auf die Hochschulthemen, die zweite, zeitlich etwas kürzere auf die Schulthemen. Beide Abendveranstaltungen bestanden aus jeweils drei Teilen. Im ersten Teil trugen informierte Teilnehmer unserer Arbeitsgruppe als »Experten« den Testpersonen die verschiedenen Standpunkte zu den einzelnen Themen vor. Anschließend setzten sich die Testpersonen in zufällig ausgelosten Kleingruppen zusammen und deliberierten über die Themen unter Leitung eines Moderators aus unserer Arbeitsgruppe. Der dritte Teil bestand aus einer weiteren Plenarsitzung, in der die einzelnen Kleingruppen den »Experten« Fragen stellen konnten. Aufgrund der begrenzten Teilnehmerzahl und der Veranstaltungsform der Schülerakademie war es nicht möglich, zusätzlich eine Kontrollgruppe an Testpersonen zu untersuchen, die nicht an der Deliberationsphase teilnahmen.

Wie oben angedeutet, galt unser Interesse vor allem dem Prozess und weniger den im Prozess generierten Umfragedaten, insbesondere weil diese Daten aufgrund der Zusammensetzung der Befragten nicht repräsentativ für eine größere Referenzbevölkerung sind. Dennoch kommt, wie wir nun zeigen, den Daten eine Schlüsselbedeutung zu: Sie eignen sich zur quantitativen Beurteilung des Deliberationsprozesses.

Quantitative Kriterien zur Beurteilung des Deliberationsprozesses waren:

- der Informationsstand der Befragten nach der Deliberation im Vergleich zu vorber, 
- die kollektive Kohäsion der Präferenzen der unterschiedlichen Befragten in Form des Maßes des Metakonsenses nach der Deliberation im Vergleich zu vorher.

Den Einfluss der Deliberation auf den Informationsstand der Befragten untersuchten wir anhand von zwei Wissensfragen:

- Frage 2.2: Findet an Universitäten in den USA ein leistungsbezogenes Auswahlverfahren statt? (Die korrekte Antwort hier lautet »Ja«.)

- Frage 5.2: In welchen Bundesländern in Deutschland gibt es bereits das Zentralabitur? (Die vollständige korrekte Liste hier lautet »Bayern, Baden-Württemberg, Saarland, Thüringen, Sachsen, Sachsen-Anhalt, Mecklenburg-Vorpommern«.)

Darstellung 2 fasst die Ergebnisse zu den Wissensfragen zusammen.

\section{Darstellung 2}

Frage 2.2:

Anzahl der Befragten mit korrekter Antwort vor der Deliberation: 46 (49\%) Anzahl der Befragten mit korrekter Antwort nach der Deliberation: 75 (81\%)

\section{Frage 5.2:}

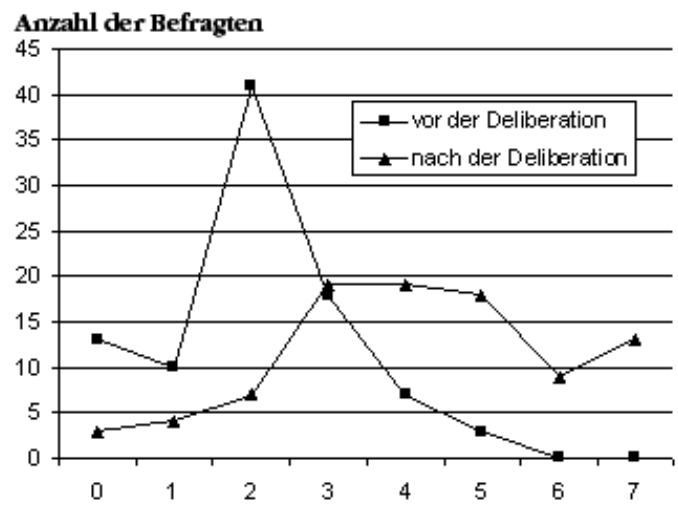

Anzahl der korrekten Antworten

Die Ergebnisse zeigen, dass Informationsfragen nach der Deliberationsphase korrekter beantwortet wurden als vor der Deliberationsphase. Zu Frage 2.2 stieg der Prozentsatz der Befragten mit korrekter Antwort von 49\% vor der Deliberation auf $81 \%$ nach der Deliberation. Zu Frage 5.2 stieg die durchschnittliche Anzahl korrekt genannter Bundesländer mit Zentralabitur von 2 vor der Deliberation auf 4 nach der Deliberation. Dies unterstützt die Hypothese, dass Deliberation zum Lernen von Informationen führen kann.

Den Einfluss der Deliberation auf das Maß des Metakonsenses unter den Befragten untersuchten wir anhand von Präferenzfragen zu mehreren Themen: Studienfinanzierung (Frage 1.1), Zahlungsform von eventuellen Studiengebühren (Frage 1.2), Hochschulzugang (Frage 2.1), mehrgliedrige Schule versus Gesamtschule (Frage 3), Halbtagsschule versus Ganztagsschule (Frage 4.1), Lokal- versus Zentralabitur 
(Frage 5.1). Zu jeder Präferenzfrage konnten die Befragten verschiedene Alternativmodelle in eine Präferenzrangfolge (von der höchsten bis zur niedrigsten Präferenz) setzen. Für Details verweisen wir auf den im Anhang abgedruckten Fragebogen.

Zur Quantifikation des Maßes des Metakonsenses vor und nach der Deliberation ermittelten wir zu beiden Zeitpunkten zu jeder einzelnen Präferenzfrage den Anteil der Befragten - in Prozent - mit eingipfligen Präferenzen in Bezug auf dieselbe Links-Rechts-Achse. Wir ermittelten außerdem, welcher Links-Rechts-Anordnung der Alternativen diese Achse entsprach (jeweils vor und nach der Deliberation), sowie den jeweiligen Condorcet-Sieger (ebenfalls jeweils vor und nach der Deliberation).

Darstellung 3 fasst die Ergebnisse zu den Präferenzfragen zusammen.

Darstellung 3

\begin{tabular}{|l|c|l|l|l|c|c|}
\hline $\begin{array}{l}\text { Frage / Thema } \\
\text { (siehe Fragen- } \\
\text { bogen) }\end{array}$ & \multicolumn{2}{|l|}{$\begin{array}{l}\text { Prozent der } \\
\text { Befragten } \\
\text { (aus einer Gesamt- } \\
\text { zahl von 93) mit } \\
\text { eingipfligen Präfe- } \\
\text { renzen auf dersel- } \\
\text { ben Links-Rechts- } \\
\text { Achse }\end{array}$} & $\begin{array}{l}\text { Links-Rechts- } \\
\text { Achse } \\
\text { (für die Inter- } \\
\text { pretation der } \\
\text { Modelle siehe Fra- } \\
\text { gebogen) }\end{array}$ & \multicolumn{2}{l|}{ Condorcet-Sieger } \\
\cline { 2 - 7 } & $\begin{array}{l}\text { vor der } \\
\text { Delibe- } \\
\text { ration }\end{array}$ & $\begin{array}{l}\text { nach der } \\
\text { Delibe- } \\
\text { ration }\end{array}$ & $\begin{array}{l}\text { vor der } \\
\text { Delibe- } \\
\text { ration }\end{array}$ & $\begin{array}{l}\text { nach der } \\
\text { Delibe- } \\
\text { ration }\end{array}$ & $\begin{array}{l}\text { vor der } \\
\text { Delibe- } \\
\text { ration }\end{array}$ & $\begin{array}{l}\text { nach der } \\
\text { Delibe- } \\
\text { ration }\end{array}$ \\
\hline $\begin{array}{l}\text { 1.1 Studienfinan- } \\
\text { zierung }\end{array}$ & $43 \%$ & $52 \%(+)$ & $\begin{array}{l}\text { C A B D } \\
\text { E }\end{array}$ & $\begin{array}{l}\text { C A B D } \\
\text { E }\end{array}$ & B & B \\
\hline 1.2 Zahlungsform & $83 \%$ & $87 \%(+)$ & A B C & A B C & B & B \\
\hline $\begin{array}{l}\text { 2.1 Hochschul- } \\
\text { zugang }\end{array}$ & $63 \%$ & $67 \%(+)$ & A C D B & A D C B & D & D \\
\hline $\begin{array}{l}\text { 3. Mehrgliedrige } \\
\text { Schule versus } \\
\text { Gesamtschule }\end{array}$ & $90 \%$ & $80 \%(-)$ & A B C D & B A C D & A & A \\
\hline $\begin{array}{l}\text { 4.1 Ganztags- } \\
\text { schule }\end{array}$ & $95 \%$ & $96 \%(0)$ & A B C & A B C & B & B \\
\hline $\begin{array}{l}\text { 5.1 Lokal- versus } \\
\text { Zentralabitur }\end{array}$ & $91 \%$ & $87 \%(-)$ & A B C & A B C & C & C \\
\hline
\end{tabular}

Legende: $(+)=$ Anstieg des Maßes an partieller Eingipfligkeit

$(-)=$ Rückgang des Maßes an partieller Eingipfligkeit

$(0)=$ keine wesentliche Veränderung des Maßes an partieller Eingipfligkeit 
Die Ergebnisse zu den Hochschulthemen (Fragen 1.1, 1.2 und 2.1) unterstützen die Hypothese, dass Deliberation das Maß an partieller Eingipfligkeit vergrößern kann, während die Ergebnisse zu den Schulthemen (Fragen 3, 4.1 und 5.1) diese Hypothese nicht unterstützen.

Der Anstieg des Maßes an partieller Eingipfligkeit bei den Hochschulthemen, der einen Anstieg des Metakonsenses vermuten lässt, ist möglicherweise dadurch zu erklären, dass der Kenntnis- und Meinungsstand der befragten Schüler zu Themen der Hochschulpolitik vor der Deliberation vergleichsweise gering und unstrukturiert war und der Deliberationsprozess daher zu einer Präferenzstrukturierung führen konnte. Der eher gegenläufige Effekt bei den Schulthemen könnte möglicherweise daraus resultieren, dass die befragten Schüler schon vor der Deliberation starke (und darüber hinaus sehr homogene, zum Teil fast einstimmige) Meinungen zu Themen der Schulpolitik vertraten und der Deliberationsprozess zu einer gewissen »Aufrüttelung « führte, z. B. durch die Begegnung mit vorher kaum bekannten Standpunkten zur Gesamtschule. Die Deliberation änderte außerdem die dominante Links-Rechts-Achse der Alternativen zur Struktur des Schulsystems. Vor der Deliberation wurde das Mischsystem (Modell B) (mehrgliedriges Schulsystem mit Gesamtschule als Option) als eine in der Mitte liegende Kompromisslösung eingeordnet (A B C D). Nach der Deliberation hingegen rückte dieses an den Rand der Links-Rechts-Achse (B A C D). Diese Veränderung der Links-Rechts-Achse ist möglicherweise auf die im Deliberationsprozess geführte Argumentation zurückzuführen, dass die Gesamtschule im Rahmen eines Mischsystems (also im Modell B) zu schlechteren Ergebnissen führt als in einem System mit der Gesamtschule als einziger Schulform.

Ein anderer Faktor, der zu den abweichenden Ergebnissen zwischen Schul- und Hochschulthemen beigetragen haben könnte, ist, dass aufgrund der unterschiedlichen Länge der beiden Abendveranstaltungen weniger Zeit der Deliberation für die Schulthemen als für die Hochschulthemen zur Verfügung stand.

Wie wir erläutert haben, unterschied sich unser Deliberationsprojekt von Fishkins Deliberative Polls dadurch, dass es in einem Bildungszusammenhang durchgeführt wurde und die Gruppe der Befragten keine repräsentative Stichprobe aus einer entsprechenden Referenzbevölkerung war. Dennoch sind unsere empirischen Ergebnisse zu den Auswirkungen der Deliberation auf die zwei untersuchten quantitativen Kriterien - Informationsstand und Maß des Metakonsenses - mit den Ergebnissen aus Fishkins Deliberative Polls konsistent. Wie in der Studie von Luskin, Fishkin und Jowell konnten wir einen Anstieg des Informationsstandes nachweisen, und wie in der Studie von List, McLean, Fishkin und Luskin konnten wir zumindest für einen Teil der Präferenzfragen einen Anstieg der partiellen Eingipfligkeit nachweisen ${ }^{22}$.

22 Siehe Luskin / Fishkin / Jowell, aaO. (FN 18); List / McLean / Fishkin / Luskin, aaO. (FN 19). 


\section{Schlussfolgerungen}

Die Ergebnisse unseres Deliberationsprojekts deuten darauf hin, dass sich die Methode des Deliberative Polling zur Förderung demokratischer Kompetenzen in mehrerlei Hinsicht eignet:

- Das Deliberative Polling bildet sowohl aggregative als auch deliberative Aspekte demokratischer Prozesse ab und lässt einen »Mikrokosmos « entstehen, in dem die Teilnehmer durch persönliche Erfahrung ein Verständnis für reale demokratische Kommunikations- und Entscheidungsprozesse entwickeln können. Insbesondere kann der deliberative Aspekt die Fähigkeit zur argumentativen Begründung politischer Standpunkte fördern.

- Das Deliberative Polling kann zu einem Anstieg des Informationsstands der Teilnehmer führen und somit, zumindest in der Gruppe der Befragten, einer Situation der »rationalen Ignoranz « entgegenwirken.

- Das Deliberative Polling kann Problemfelder strukturieren, zu einem Anstieg des Metakonsenses unter den Teilnehmern führen und somit die Lösung von Aggregationsproblemen erleichtern. Vor dem Hintergrund der Diagnose, dass Aggregationsprobleme wie Condorcet-Paradoxien auf einen Mangel an Struktur in den Präferenzinputs zurückzuführen sind, kommt den durch Deliberation angeregten Strukturierungsprozessen eine wichtige instrumentelle Bedeutung zur Ermöglichung konsistenter Aggregation zu.

\section{Anhang: Fragebogen: Aktuelle Fragen der deutschen Bildungspolitik}

\section{Studienfinanzierung}

1.1 Betrachten Sie die folgenden Alternativvorschläge zum Thema Studienfinanzierung:

Modell A grundsätzlich keine Studiengebühren

Modell B Studiengebühren erst ab einer bestimmten Semesterzahl (z. B. für Langzeitstudierende oder für Aufbaustudiengänge)

Modell C Studiengebühren ab dem 1. Semester, aber mit staatlichen Stipendien, die primär nach sozialen/finanziellen Kriterien vergeben werden

Modell D Studiengebühren ab dem 1. Semester, aber mit staatlichen Stipendien, die primär nach akademischen Leistungskriterien vergeben werden

Modell E Studiengebühren nach freier marktorientierter Wahl der Hochschulen

Meine Präferenzen über die Modelle sind: (Bitte jeweiligen Buchstaben des Modells angeben.)

\begin{tabular}{|l|l|l|l|l|}
\hline 1. (höchste) Präf. & 2. Präferenz & 3. Präferenz & 4. Präferenz & 5. Präferenz \\
\hline & & & & \\
\hline
\end{tabular}


1.2 Stellen Sie sich vor, Studiengebühren würden eingeführt. Betrachten Sie folgende Alternativvorschläge zur Zahlungsform:

Modell A Studiengebühren sind während des Studiums zu bezahlen; kein staatliches Finanzierungsmodell

Modell B Studiengebühren sind erst nach dem Studium zu bezahlen; Studierende erhalten einen Kredit mit günstigen Konditionen

Modell C Studiengebühren sind erst nach dem Studium zu bezahlen durch eine am Einkommen orientierte Steuer (d. h. hohe Rückzahlung für hohes Einkommen; niedrige Rückzahlung für niedriges Einkommen).

Meine Präferenzen über die Modelle sind: (Bitte jeweiligen Buchstaben des Modells angeben.)

\begin{tabular}{|l|l|l|}
\hline 1. (höchste) Präf. & 2. Präferenz & 3. Präferenz \\
\hline & & \\
\hline
\end{tabular}

\section{Hochschulzugang}

2.1 Betrachten Sie folgende Alternativvorschläge zu den Zulassungskriterien zum Studium an einer Hochschule:

Modell A keinerlei Zugangsbeschränkung zum Studium an einer Hochschule; freie Einschreibung nach Bestehen des Abiturs

Modell B einziges Zulassungskriterium ist der Notendurchschnitt im Abitur (Numerus Clausus)

Modell C Auswahl der Studierenden durch die Universitäten nach fachbezogenen kognitiven Leistungstests (z. B. schriftliche Aufnahmeprüfungen)

Modell D Auswahlverfahren der Studierenden durch die Universitäten mit Hilfe von schriftlichen Bewerbungen und persönlichen Gesprächen Meine Präferenzen über die Modelle sind: (Bitte jeweiligen Buchstaben des Modells angeben.)

\begin{tabular}{|l|l|l|l|}
\hline 1. (höchste) Präf. & 2. Präferenz & 3. Präferenz & 4. Präferenz \\
\hline & & & \\
\hline
\end{tabular}

2.2 Findet an Universitäten in den USA ein leistungsbezogenes Auswahlverfahren statt?

$\mathrm{Ja}$

Nein

Weiß nicht 


\section{Struktur des Schulsystems}

Modell A ausschließlich das bestehende zwei- bzw. dreigliedrige Schulsystem (Gymnasium, (Real-) und Hauptschule, je nach Bundesland unterschiedlich)

Modell B das bestehende zwei- bzw. dreigliedrige Schulsystem mit der Gesamtschule als Alternativangebot

Modell C ausschließlich Gesamtschulen mit Binnendifferenzierung (d.h. Schüler werden in verschiedenen leistungsdifferenzierten Kursen unterrichtet)

Modell D ausschließlich Gesamtschulen ohne Binnendifferenzierung (d.h. alle Schüler werden in gemeinsamen Kursen unterrichtet)

Meine Präferenzen über die Modelle sind: (Bitte jeweiligen Buchstaben des Modells angeben.)

\begin{tabular}{|l|l|l|l|}
\hline 1. (höchste) Präf. & 2. Präferenz & 3. Präferenz & 4. Präferenz \\
\hline & & & \\
\hline
\end{tabular}

\section{Halbtags- versus Ganztagsschule}

4.1 Betrachten Sie folgende Alternativvorschläge:

Modell A eine für alle Schüler verpflichtende Ganztagsschule

Modell B freie Entscheidungsmöglichkeit der Eltern und Schüler zwischen Ganztagsschule und konventionellen (Halbtags-)Schulen

Modell C grundsätzlich keine Ganztagsschule

Meine Präferenzen über die Modelle sind: (Bitte jeweiligen Buchstaben des Modells angeben.)

\begin{tabular}{|l|l|l|}
\hline 1. (höchste) Präf. & 2. Präferenz & 3. Präferenz \\
\hline & & \\
\hline
\end{tabular}

4.2 Stellen Sie sich vor, die verpflichtende Ganztagsschule würde eingeführt. Wie stehen Sie zu den folgenden Vorschlägen zur Gestaltung des Nachmittagsunterrichts? (Befürwortung mehrerer Vorschläge ist in dieser Frage möglich.)

\begin{tabular}{|l|l|l|l|}
\hline & lehne ich ab & $\begin{array}{l}\text { keine } \\
\text { Meinung }\end{array}$ & $\begin{array}{l}\text { befürworte } \\
\text { ich }\end{array}$ \\
\hline $\begin{array}{l}\text { Freizeitangebote am Nachmittag, } \\
\text { wie sportliche oder musische Akti- } \\
\text { vitäten }\end{array}$ & & & \\
\hline $\begin{array}{l}\text { Individualförderung am Nachmit- } \\
\text { tag, sowohl für leistungsschwä- } \\
\text { chere als auch für begabtere Schüler }\end{array}$ & & & \\
\hline
\end{tabular}




\begin{tabular}{|l|l|l|l|}
\hline $\begin{array}{l}\text { verpflichtendes soziales Engage- } \\
\text { ment am Nachmittag (beispielswei- } \\
\text { se in Form von Projekten) }\end{array}$ & & & \\
\hline $\begin{array}{l}\text { konventioneller Schulunterricht am } \\
\text { Nachmittag genau wie bisher am }\end{array}$ & & & \\
Vormittag & & & \\
\hline
\end{tabular}

\section{Abiturprüfungen}

5.1 Betrachten Sie folgende Alternativvorschläge:

Modell A überhaupt kein Zentralabitur - schulinterne Abiturprüfungen

Modell B landesweites Zentralabitur

Modell C bundesweites Zentralabitur

Meine Präferenzen über die Modelle sind: (Bitte jeweiligen Buchstaben des Modells angeben.)

\begin{tabular}{|l|l|l|}
\hline 1. (höchste) Präf. & 2. Präferenz & 3. Präferenz \\
\hline & & \\
\hline
\end{tabular}

5.2 In welchen Bundesländern in Deutschland gibt es bereits das Zentralabitur: 